\title{
Neumonía organizada como forma de presentación de una artritis reumatoide
}

\section{Organizing pneumonia as the presenting symptom of rheumatoid arthritis}

\author{
Verónica Temprado, Laura Manzanedo, Gloria Alonso, Antonio Chamorro \\ Servicio de Medicina Interna. Hospital Clínico de Salamanca. SACYL Salamanca
}

\section{Resumen}

La neumonía organizada (NO) es una enfermedad pulmonar intersticial que puede asociarse a la artritis reumatoide (AR). Normalmente, la afectación pulmonar se presenta tras años de evolución de las manifestaciones articulares, siendo menos frecuente la aparición concomitante de ambos procesos. Se expone el caso de una paciente de 79 años que presentó tos y disnea junto con inflamación articular y criterios serológicos de AR. La radiografía de tórax (Rx) y la tomografía axial computerizada (TAC) objetivaron un infiltrado alveolar bibasal con broncograma aéreo. Las manifestaciones pulmonares severas de una NO motivaron el ingreso y fueron la clave para el diagnóstico final de una AR. La paciente recibió tratamiento corticoideo con excelente respuesta.

Palabras clave: Artritis Reumatoide, neumonía organizada, síntomas

\section{Introducción}

La AR es la artropatía inflamatoria más común, afecta al 1\% de la población mundial'. Se caracteriza por alteraciones inflamatorias que afectan al tejido articular y estructuras relacionadas, sin embargo tiene también carácter de enfermedad sistémica con múltiples manifestaciones extrarticulares. Una de ellas es la enfermedad pulmonar intersticial (EPI), que se presenta entre el 4 y el $68 \%$ de los pacientes con $A R^{2}$. Dentro de este grupo la forma más frecuente de presentación es la neumonía intersticial usual (NIU), seguida de la neumonía intersticial no específica (NINE)

\section{Abstract}

Organizing pneumonia (OP) is an interstitial lung disease which may be associated to rheumatoid arthritis (RA). Normally, pulmonary involvement appears after years of evolution of the joint manifestations, being the concomitant appearance of both processes less common. We present the case of a 79-year-old woman who presented with cough and dyspnea together with joint inflammation and serological criteria for RA. The thoracic $\mathrm{X}$-ray and the CAT scan revealed a bibasilar alveolar infiltrate with air bronchogram. The severe pulmonary manifestations of OP caused the hospital admission and were essential for the final diagnosis of RA. The patient received treatment with corticosteroids with an excellent response. Key words: Rheumatoid Arthritis, organizing pneumonia, symptoms.

y finalmente, la neumonía organizada (NO)³. Lo habitual es que la afectación pulmonar se presente en fases avanzadas de la enfermedad articular, siendo más raro que la lesión pulmonar preceda o coincida con el diagnóstico de AR. Presentamos el caso de una paciente con diagnóstico de NO como primera manifestación de una AR hasta el momento no diagnosticada.

\section{Caso clínico}

Mujer de 79 años sin hábitos tóxicos, con antecedentes de artrosis e intolerancia a la lactosa, con consumo de ibuprofeno ocasional. Ingresó en Medicina Interna por un cuadro de semanas de evolu-

Figura 1. Radiografía de manos: subluxaciones, reabsorción subperióstica, y erosiones marginales.

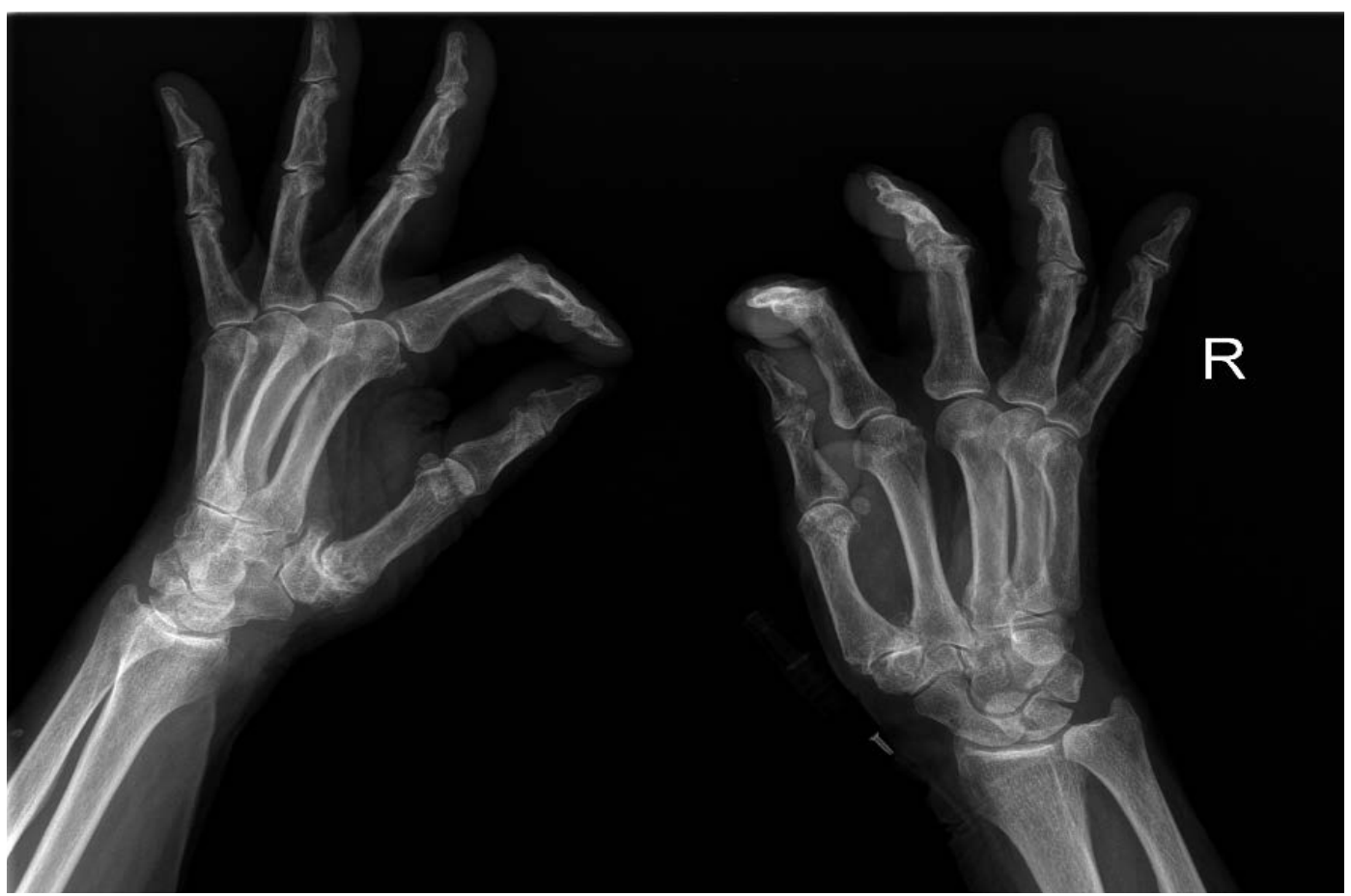




\begin{tabular}{|c|c|c|c|c|c|c|c|c|c|}
\hline \multicolumn{4}{|l|}{ Automiticos } & \multicolumn{3}{|l|}{ Hemortamy } & \multicolumn{3}{|c|}{ Ac.en artitits reumatoide } \\
\hline Gloses & 135 & $m / d h$ & $(802-115)$ & Hematies & $3970000 / \mathrm{L}$ & $(4.5,4)$ & & & \\
\hline Urea & 26 & $\mathrm{mg}_{\mathbf{g}} / \mathrm{d}$ & $(16.6-48.5)$ & Hemoglobina & $12.2 \mathrm{~g} / \mathrm{dL}$ & $(12-16)$ & $\begin{array}{l}\text { AcAnti- } \\
\text { Péctido }\end{array}$ & $117.5 \mathrm{U} / \mathrm{mL}$ & $\begin{array}{l}\text { Negenivo:0-20 } \\
\text { Postivomoderaso: 20-60 }\end{array}$ \\
\hline Creatrinin & 0.59 & $m_{8} / d t$ & (0.5-0.9) & Leucocitos & 10080 /ul. & $(45-10.8)$ & citrulinado & & Postvofuerte: $>60$ \\
\hline Sodio & 140 & $\mathrm{mmo} / \mathrm{h}$ & (135-145) & Neutrófllor & $7680 \quad / 4$. & $(14.6 .5)$ & & & \\
\hline Peassio & 49 & mmo/h & (3.5.5.1) & Linfocitos & 1130 /ul & (12-3.5) & & & \\
\hline Calclo & 9.3 & $\mathrm{mg} / \mathrm{d}$ & (88.10.2) & Plaquetas & $292000 \mathrm{JuL}$ & $(150-450)$ & Factor & $19.6 \mathrm{U} / \mathrm{mL}$ & $(0.1 \cdot 14)$ \\
\hline $\begin{array}{l}\text { Proteinas totales } \\
\text { Bi toctal }\end{array}$ & 6.6 & $\begin{array}{c}\mathrm{g} / \mathrm{d} . \\
\mathrm{m} / \mathrm{dt}\end{array}$ & $(6.4 .83)$ & Proteinograma sérico & 0 & & reumatoide & & \\
\hline AST & 26 & uh & $(0.32)$ & Albúmina & $38.3 \%$ & (55-66.1) & & & \\
\hline ATT & 26 & un & (0.-33) & Alfa-1 Globulinas & $8.10 \%$ & $(2.9-4.9)$ & & & \\
\hline BA & 119 & un & (35.:105) & Alfa.2 Globulinas & $13.7 \%$ & $(7.1 \cdot 11.8)$ & & & \\
\hline GGा & 71 & un & $(0.00)$ & Beta Globulinas & $16.3 \%$ & (8.4-13.1) & & & \\
\hline LOH & 279 & un & $(135.214)$ & Gamma Globulinas & $23.6 \%$ & [11.1-18.8] & & & \\
\hline PCR & 10.73 & $\mathrm{~m} / \mathrm{d}$ & $(0.05)$ & Albúmina & $2.68 \mathrm{~g} / \mathrm{del}$ & $(4.02 \cdot 4.76)$ & & & \\
\hline Metaboligmo del iverro & & & & Alth.1 Globulinas & $0.57 \mathrm{~g} / \mathrm{d}$ & $(0.21 \cdot 0.35)$ & & & \\
\hline Hierro & $x$ & ug/d & (37-145) & Alis-2 Globulinas & $0.96 \mathrm{~g} / \mathrm{dd}$ & $(0.51 \cdot 0.085)$ & & & \\
\hline Trarsferrina & 138 & $\mathrm{mg} / \mathrm{dt}$ & $(200-360)$ & Beta Globulinas & $1.14 \mathrm{~g} / \mathrm{dl}$ & $(.60 .0 .94)$ & & & \\
\hline Ferritina & 465 & $\mathrm{rg} / \mathrm{mb}$ & $(30-900)$ & Gamma Globulinas & $1.65 \mathrm{~g} / \mathrm{dl}$ & $(.80-1.35)$ & & & \\
\hline CapToral de fijacion & 175 & ut/dt & $(260 .-388)$ & Proteinas plasmáticas & & & & & \\
\hline Indce de staraclión & 16.6 & $\mathrm{x}$ & $(16-45)$ & $\begin{array}{l}\text { Inmuncolobulina G } \\
\text { Inmuncglobulina A }\end{array}$ & $\begin{array}{l}1700 \mathrm{mg} / \mathrm{dl} \\
556 \mathrm{mg} / \mathrm{dl}\end{array}$ & $\begin{array}{r}(751-1560) \\
(82-453)\end{array}$ & & & \\
\hline v56 & 112 & $m m$ & $(1.25)$ & Inmunoglobulina M & $26.1 \mathrm{mg} / \mathrm{dl}$ & $(46-304)$ & & & \\
\hline
\end{tabular}

ción de tos con expectoración amarillenta y disnea sin fiebre. En la anamnesis dirigida la paciente contaba rigidez articular matutina en manos junto con episodios de artritis metacarpo falángicas y en codos. En la exploración física destacaba una auscultación pulmonar con crepitantes gruesos bibasales y la exploración de extremidades evidenció una desviación articular cubital, edema en dedos de las manos, artritis de muñecas y codo izquierdo. La analítica se resume en la tabla 1. La Rx de tórax mostró áreas de condensación bibasal con broncograma aéreo de predominio derecho. Se inició antibioterapia empírica con ceftriaxona y levofloxacino sin mejoría. Todos los estudios microbiológicos y de autoinmunidad resultaron negativos, excepto el factor reumatoide que fue positivo: $19.6 \mathrm{UI} /$ $\mathrm{mL}$ (0.1-14) así como los anti péptido citrulinado (anti PPC), 117.5 $\mathrm{UI} / \mathrm{mL}$ (positivo fuerte $>60$ ).

La Rx de manos evidenció subluxaciones, reabsorción subperióstica, y erosiones marginales. (Figura 1).

Se realizó fibrobroncoscopia, la citología del aspirado bronquial y lavado bronquial reveló un componente inflamatorio agudo con presencia de abundantes linfocitos y monocitos (microbiología negativa y citología negativa para células malignas). No pudo realizarse biopsia transbronquial porque la paciente toleró mal la prueba.

Se realizó una TC torácica en la que se objetivaron áreas parcheadas bilaterales de aumento de densidad en vidrio deslustrado de predominio periférico y subpleural compatible con NO. (Figura 1). Se instauró tratamiento corticoideo a dosis de $1 \mathrm{mg} / \mathrm{Kg}$ de peso con desaparición de la clínica y normalización del perfil hepático y los reactantes de fase aguda. EI TACAR de control a las 10 semanas informó de mejoría radiológica.

\section{Discusión}

La AR de inicio en mayores de 65 años se conoce con el nombre de elderly onset rheumatoid arthritis (EORA). La principal diferencia con respecto a los jóvenes es que aparece por igual en hombres que en mujeres y que clínicamente tiene una forma de presentación más aguda, como un cuadro agudo sistémico, con intenso dolor en grandes articulaciones proximales, acompañado de astenia y pérdida de peso, y por otro lado, con mayor afectación sistémica como las manifestaciones pulmonares. Para su diagnóstico debemos aplicar los criterios de EULAR y ACR ${ }^{4}$.

La EPI es una manifestación extrarticular que se presenta según las series entre 4 y $68 \%$ de los pacientes ${ }^{5,6}$. Dentro de la EPI asociada a la AR, las más frecuentes son la NIU y la NINE, siendo menos habitual la asociación con NO que, además, tiene mejor pronóstico que las anteriores. Lo habitual es que la enfermedad pulmonar se presente años después del diagnóstico de la $A R$, pero ocasionalmente el diagnóstico de la NO precede o es simultáneo al de la AR. En una revisión de 26 casos publicados entre 1987 y 2006 de NO asociada a AR, Mori et al encontraron que casi en un $81 \%$ el diagnóstico fue posterior, en el $15 \%$ precedió y en el $5 \%$ fue concomitante al diagnóstico de $A R^{7}$. En los casos revisados, la mayoría de los pacientes eran mayores de 65 años, por lo que parece que la afectación pulmonar es más frecuente que en los jóvenes. 


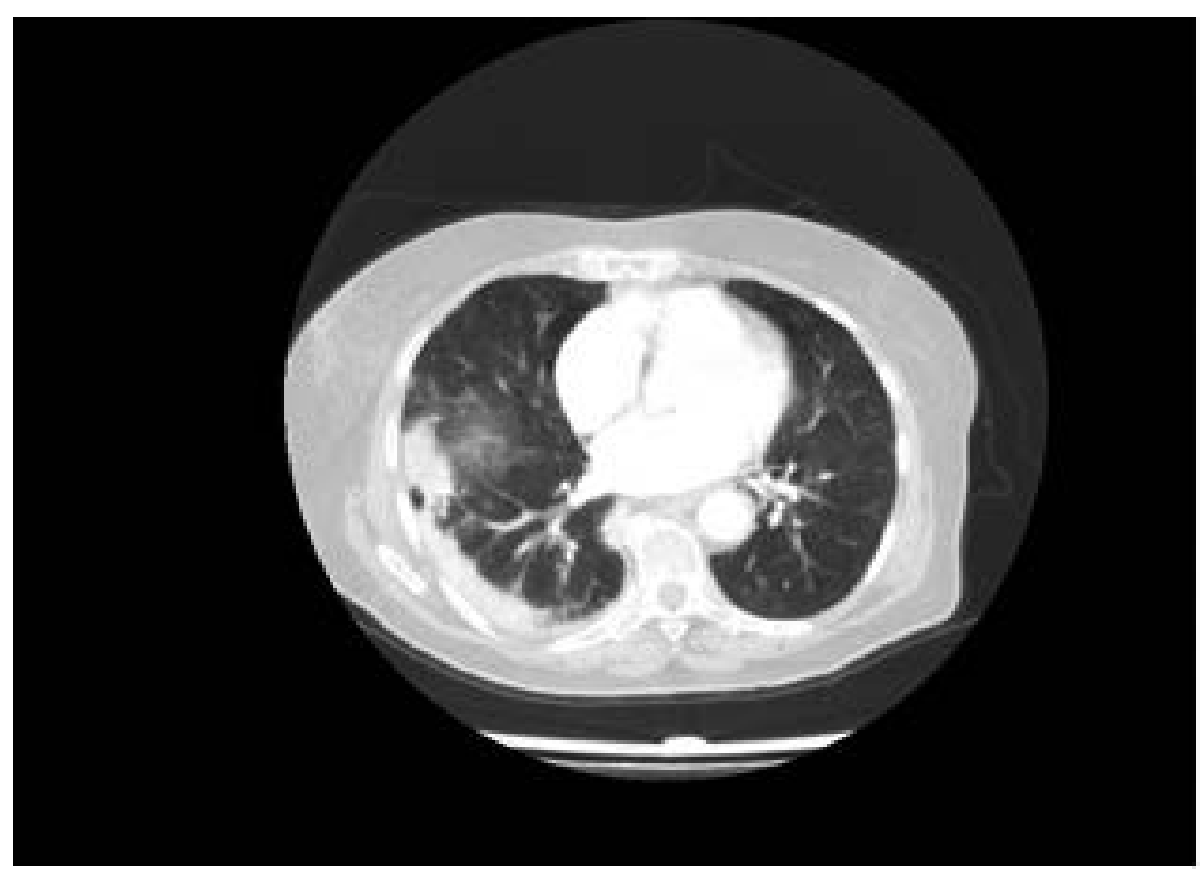

En la NO la TAC torácica muestra las características opacidades subpleurales en vidrio deslustrado ${ }^{8}$. El diagnóstico definitivo es histopatológico, en la mayoría de casos por biopsia transbronquial o videotoracoscopia, demostrándose fibroblastos en los bronquiolos y alvéolos ${ }^{9}$.

El objetivo del tratamiento de la AR en los pacientes ancianos es el mismo que en los de menor edad y consiste en conseguir el control de los síntomas y la remisión de la enfermedad y para ello contamos con los mismos fármacos. Los AINES asocian mayores efectos secundarios y deben evitarse en la medida de lo posible. Tradicionalmente se han usado la corticoides como base fundamental del tratamiento, pero actualmente los fármacos modificadores de la enfermedad como el metotrexate han demostrado que son igual de efectivos y seguros que entre los más jóvenes si la función renal esta conservada. Y lo mismo se puede decir respecto a los antiTNF ${ }^{1}$ Respecto a el pronóstico de la NO asociada a AR es generalmente favorable con respuesta rápida a los corticoides en dosis de $0.75-1.5 \mathrm{mg} / \mathrm{Kg} / \mathrm{día}$. Si no ocurre respuesta se podrían emplear la azatioprina o la ciclofosfamida como ahorradores de corticoides ${ }^{10}$.

\section{Conclusiones}

La AR debe considerarse en el diagnóstico diferencial de los pacientes diagnosticados de NO. Excluidas otras causas de neumonía, unas manifestaciones clínico-radiológicas sugerentes de NO junto con clínica y análisis serológicos compatibles con AR pueden resultar suficientes para iniciar tratamiento corticoideo sin necesidad de realizar biopsia pulmonar, especialmente en los casos de pacientes mayores con mala tolerancia o imposibilidad de realización de fibrobroncoscopia o toracoscopia.

\section{Bibliografía}

1. Gabriel SE. The epidemiology of rheumatoid arthritis. Rheum Dis Clin North Am 2001;27:269-81.

2. Kim EJ, Collard HR, King TE, Jr. Rheumatoid arthritis-associated interstitial lung disease: the relevance of histopathologic and radiographic pattern. Chest 2009;136:1397-405.

3. de Lauretis A, Veeraraghavan S, Renzoni E. Review series: Aspects of interstitial lung disease: connective tissue disease-associated interstitial lung disease: how does it differ from IPF? How should the clinical approach differ? Chron Respir Dis 2011;8:53-82.

4. García Arias MJ, García Vadillo A.Tratamiento de la artritis reumatoide del anciano. Semin Fundamental Esp Reumatol. 2011;12:103-107.

5. Gutsche M, Rosen GD, Swigris JJ. Connective Tissue Disease-associated Interstitial Lung Disease: A review. Curr Respir Care Rep 2012;1:224-32.

6. American Thoracic Society/European Respiratory Society International Multidisciplinary Consensus Classification of the Idiopathic Interstitial Pneumonias. This joint statement of the American Thoracic Society (ATS), and the European Respiratory Society (ERS) was adopted by the ATS board of directors, June 2001 and by the ERS Executive Committee, June 2001. Am J Respir Crit Care Med 2002;165:277-304.

7. Mori S, Cho I, Koga Y, Sugimoto M. A simultaneous onset of organizing pneumonia and rheumatoid arthritis, along with a review of the literature. Mod Rheumatol 2008;18:60-6.

8. Remy-Jardin M, Remy J, Cortet B, Mauri F, Delcambre B. Lung changes in rheumatoid arthritis: CT findings. Radiology 1994;193:375-82.

9. Bradley B, Branley HM, Egan JJ, et al. Interstitial lung disease guideline: the British Thoracic Society in collaboration with the Thoracic Society of Australia and New Zealand and the Irish Thoracic Society. Thorax 2008;63 Suppl 5:v1-58.

10. Lee J, Cha SI, Park TI, Park JY, Jung TH, Kim CH. Adjunctive effects of cyclosporine and macrolide in rapidly progressive cryptogenic organizing pneumonia with no prompt response to steroid. Intern Med 2011;50:475-9. 